\title{
Fatores de risco associados a sintomas depressivos em adolescentes de um município
}

\section{de pequeno porte}

\author{
Risk factors associated with depressive symptoms in adolescents in a small municipality \\ Factores de riesgo asociados a síntomas depresivos en adolescentes de una ciudad pequeña
}

Recebido: 26/03/2021 | Revisado: 08/04/2021 | Aceito: 09/04/2021 | Publicado: 19/04/2021

Ademilson José Bernardineli

ORCID: https://orcid.org/0000-0001-6442-1339

Universidade Estadual de Maringá, Brasil

E-mail: adejb@hotmail.com

Lincoln Luis Silva

ORCID: https://orcid.org/0000-0001-8445-0743

Universidade Estadual de Maringá, Brasil

E-mail: sirlincolnls@gmail.com

Claudia Regina Marchiori Antunes Araújo

ORCID: https://orcid.org/0000-0002-7234-6604

Instituto Adventista Paranaense, Brasil

E-mail: claudia_marchiori@hotmail.com

Raíssa Bocchi Pedroso

ORCID: https://orcid.org/0000-0002-0076-1032

Universidade Estadual de Maringá, Brasil

E-mail: raissap@gmail.com

\begin{abstract}
Resumo
Introdução: Estimativas da OMS (Organização Mundial da Saúde) apontam que os transtornos depressivos em adolescentes aumentam em todo mundo, indicando que $10 \%$ a $20 \%$ vivenciam problemas de saúde mental. No Brasil, estudos mostram prevalência de $13,2 \%$, sendo considerado, portanto, um problema de saúde pública. Objetivo: Rastrear a presença de sintomas depressivos e fatores de risco associados em adolescentes de um município de pequeno porte. Metodologia: Estudo transversal, descritivo e analítico com abordagem quantitativa. Os dados foram coletados em uma amostra de 324 estudantes do município de Marialva/PR - Brasil, por meio de questionário sociodemográfico e instrumento de rastreio de episódios depressivos, o PHQ - 9 (Patiente Health Questionnaire - 9). A análise ocorreu por regressão linear, matriz de correlação e análise de redes. Resultados: 64,2\% apresentaram sintomas moderado à grave, com maior prevalência em adolescentes do sexo feminino, sendo que $20,1 \%$ mostraram sintomas graves. Os fatores de risco associados foram: maior tempo de uso de celular; não frequentar parques; não praticar esportes; usar drogas lícitas; relatar diagnóstico para ansiedade e casos de depressão na família. Adolescentes que jogam no celular sozinho apresentaram 5,1 vezes mais chances de desenvolver sintomas depressivos, e os que usam drogas em grupo, 5,5 vezes mais chance. Conclusão: O estudo sugere que cidades pequenas que apresentam estruturas urbanas inadequadas e não acolhedoras podem acentuar o problema dos transtornos depressivos em adolescentes, o que indica a necessidade de políticas públicas que melhorem as perspectivas de futuro deste público.
\end{abstract}

Palavras-chave: Depressão; Adolescente; Saúde mental; Cidade pequena.

\begin{abstract}
Introduction: World Health Organization projections indicate that depressive disorders in adolescents increase worldwide, with $10 \%$ to $20 \%$ experiencing mental health problems. In Brazil, studies show an average of $13.2 \%$, thus considered a public health issue. For that, we sought to elucidate the risk factors associated with depression symptoms in adolescents who live in small cities. Methodology: Cross-sectional, descriptive and analytical study with a quantitative approach. The data was collected from a sample of 324 students from the municipality of Marialva / PR Brazil, through a sociodemographic questionnaire and a screening tool for depressive episodes, the PHQ - 9 (Patiente Health Questionnaire - 9). The analysis occurred by linear regression, correlation matrix and network analysis. Results: $64.2 \%$ had moderate to severe symptoms, with a higher prevalence in female adolescents, with $20.1 \%$ showing severe symptoms. The associated risk factors were longer use of cell phones; not going to parks; not playing sports; using legal drugs; and reporting anxiety diagnosis and family depression cases. Adolescents who play on their cell phone alone were 5.1 times more likely to develop depressive symptoms, and those who use drugs in groups, 5.5 times. The strongest negative connections were found between PHQ-9 and being female, not going to parks or areas of sports and leisure, and reporting family depression. Conclusion: The study suggests that small cities with
\end{abstract}


inadequate and unfriendly urban structures may highlight the issue of depressive disorders in adolescents, which indicates the need for public policies to improve the prospects of this demographic.

Keywords: Depression; Adolescent; Mental health; Small town.

\begin{abstract}
Resumen
Introducción: Las estimaciones de la OMS (Organización Mundial de la Salud) indican que los trastornos depresivos en los adolescentes aumentan en todo el mundo, lo que indica que del $10 \%$ al $20 \%$ experimenta problemas de salud mental. En Brasil, los estudios muestran una prevalencia del 13,2\%, considerándose, por tanto, un problema de salud pública. Objetivo: rastrear la presencia de síntomas depresivos y factores de riesgo asociados en adolescentes de una ciudad pequeña. Metodología: Estudio transversal, descriptivo y analítico con enfoque cuantitativo. Los datos fueron recolectados en una muestra de 324 estudiantes del municipio de Marialva / PR - Brasil, a través de un cuestionario sociodemográfico e instrumento de cribado de episodios depresivos, el PHQ - 9 (Cuestionario de Salud del Paciente 9). El análisis se realizó mediante regresión lineal, matriz de correlación y análisis de redes. Resultados: 64,2\% presentaba síntomas de moderados a severos, con mayor prevalencia en las adolescentes, y el $20,1 \%$ presentaba síntomas severos. Los factores de riesgo asociados fueron: uso prolongado de teléfonos celulares; no ir a los parques; no practicar deportes; el uso de drogas legales; Informar el diagnóstico de ansiedad y casos de depresión en la familia. Los adolescentes que juegan solo con sus teléfonos móviles tienen 5,1 veces más probabilidades de desarrollar síntomas depresivos, y los que usan drogas en grupos, 5,5 veces más probabilidades. Conclusión: El estudio sugiere que las ciudades pequeñas que presentan estructuras urbanas inadecuadas y hostiles pueden acentuar el problema de los trastornos depresivos en los adolescentes, lo que indica la necesidad de políticas públicas que mejoren las perspectivas de futuro de este público.
\end{abstract}

Palabras clave: Depresión; Adolescente; Salud mental; Ciudad pequeña.

\title{
1. Introdução
}

A depressão é um transtorno mental que afeta mais de 264 milhões de pessoas no mundo, sendo caracterizada por tristeza persistente, falta de interesse ou prazer em atividades anteriormente gratificantes ou agradáveis (Organização Mundial da Saúde, 2020). Em adolescentes, estimativas da OMS apontam que o transtorno depressivo tem aumentado exponencialmente em todo o mundo.

Embora sejam susceptíveis à momentos depressivos causados pelas transformações próprias desta fase da vida, 10\% a $20 \%$ dos adolescentes vivencia problemas de saúde mental, sendo o suicídio advindo da depressão, a terceira causa de morte nesta faixa etária (OMS, 2018). No Brasil, estudos apontam que 13\% dos adolescentes têm algum diagnóstico de problema em saúde mental (Fatori, et al., 2018), com prevalência de distúrbios depressivos entre 10,2\% (Flieitlich et al., 2004) e 13,2\% (La Maison, 2018).

Fatores de risco são determinantes no desenvolvimento de sintomas depressivos em adolescentes, dentre eles, Twenge e campbell (2018) destacam o uso excessivo de smartphones e jogos eletrônicos, Bai, et al. (2018) o consumo de álcool e tabaco, e He, Parksarian e Merikangas (2018), a falta de atividade física, e Machado, et al. (2018) apontam maior prevalência para o sexo feminino. Fatores sociodemográficos como renda, baixa escolaridade, alimentação pobre em nutrientes e vulnerabilidades sociais potencializam o desenvolvimento dos transtornos mentais, bem como condições hereditárias, exposição a substâncias tóxicas e uso exagerado das tecnologias (OMS, 2018).

Com realidades diferentes dos grandes centros, o Instituto Brasileiro de Geografia e Estatística (IBGE), aponta que no Brasil há 5.132 (92,21\%) municípios considerados de pequeno porte (menos de 50.000 habitantes), e abrigam $40 \%$ da população. Considerando as relações identitárias e subjetivas que o espaço urbano imprime em seus habitantes, bem como a precariedade das condições socioeconômicas da população e a dificuldade de acesso ao sistema de saúde de muitos desses municípios, os adolescentes ocupam posição de destaque neste cenário, uma vez que podem percebem reduzidas suas expectativas com relação ao futuro, assim como as condições necessárias para o bem realizar-se.

Considerando, portanto, a problemática da depressão em adolescentes, as diversas realidades sociodemográficas, os fatores de risco desencadeantes e a escassez de estudos referentes à saúde mental em cidades pequenas, o objetivo deste estudo 
foi rastrear a presença de sintomas depressivos em adolescentes de um município de pequeno porte do Noroeste do Paraná Brasil, e identificar os fatores de risco associados.

\section{Metodologia}

Estudo transversal, retrospectivo, descritivo e analítico com abordagem quantitativa. Pereira, et al. (2018), esclarecem que que nos métodos quantitativos, faz-se a coleta de dados quantitativos ou numéricos por meio do uso de medições de grandezas e obtém-se por meio da metrologia, números com suas respectivas unidades. A coleta de dados foi realizada no ano de 2019, em todas as escolas com Ensino Fundamental e Médio do município de Marialva, região Noroeste do Paraná, que de acordo com o IBGE, abriga uma população de 31.959 habitantes. Foram pesquisados alunos em idade escolar (13 a 17 anos), matriculados no Ensino Fundamental II e Ensino Médio, totalizando 2.040 alunos.

O município possui 7 escolas de ensino fundamental II e médio, sendo 3 localizadas na região central da cidade, 2 em áreas periféricas e 2 em distritos rurais. Destas, uma é particular e as demais públicas estaduais. Em se tratando de locais de lazer e prática esportiva, o município apresenta 14 locais classificados de A1 até A14 (Tabela 1). Esta temática foi definida com base em diversos estudos que associam estes espaços com uma boa condição de saúde mental.

Tabela 1: Áreas de lazer, convivência e prática de esportes da cidade de Marialva/PR, Brasil.

\begin{tabular}{ccc}
\hline Código & Descrição & Público/Privado \\
\hline A1 & Pista de skate & Público \\
A2 & Ginásio de esportes & Público \\
A3, A8 e A10 & Pistas de caminhada & Público \\
A5 & Estádio municipal & Público \\
A11, A12, A13 e A14 & Campos de futebol & Público \\
A4 e A6 & Academias de esportes & Privado \\
A9 & Clube de recreação* & Privado \\
\hline
\end{tabular}

Fonte: Dados da pesquisa.

O tamanho amostral mínimo foi calculado com base na precisão desejada para estimar a proporção de adolescentes com sintomas depressivos, considerando confiabilidade de 95\%. Participaram do estudo, portanto, 324 estudantes, selecionados por estratificação proporcional por escola e por turma. Os adolescentes foram selecionados aleatoriamente a partir da lista de matrícula, sendo que no caso de recusa ou ausência do aluno selecionado, o mesmo foi substituído pelo próximo da lista.

Os dados foram coletados na própria escola por meio de um instrumento para caracterização do perfil sociodemográfico, com questões de interesse para esta temática, sendo elas: renda; sexo; condição econômica; acesso à tecnologia; escolaridade dos pais; uso de substâncias tóxicas; prática de esportes e moradia. Para identificar a presença de sintomas depressivos foi utilizado o instrumento Patient Health Questionnaire - 9 (PHQ-9). Este instrumento de escreening apresenta correlação com os nove sintomas clássicos da depressão, apontados pelo manual diagnóstico e estatístico de saúde mental V (DSM-V), sendo: humor deprimido; anedonia; problemas com o sono, cansaço ou falta de energia; mudanças no apetite ou peso; sentimento de culpa ou inutilidade; problemas de concentração; sentir-se lento ou inquieto e pensamentos suicidas. Utilizando uma metodologia baseada em escores de gravidade com pontos de corte em 5, 10, 15 e 20, classificam os 
níveis de sintomas depressivos em suave (5), moderado (10), moderadamente grave (15) e grave (acima de 20). Para Malas, Plioplys e Pao (2019), o PHQ-9 é a ferramenta de triagem mais comumente usada devido a fácil conclusão e pontuação, tendo sensibilidade de $89,5 \%$, especificidade de $77,5 \%$, e custo livre.

Após revisão e codificação, os dados foram inseridos em banco de dados do software Excel 6.0. A análise estatística se deu por meio do software R (versão 3.3.6), do qual a análise de regressão logística linear foi realizada e obtidas as variáveis preditivas do modelo, tendo os valores apontados no PHQ-9 como variável dependente. Em todos os testes foi observado o número de significância de 5\% ( $p<0,05)$.

Para compreender a correlação entre as variáveis preditivas foi construída a matriz de correlação. Johnson e Wichern (2007) elucidam que esta metodologia possibilita a identificação visual das variáveis envolvidas e que se correlacionaram entre si. Foram avaliados os coeficientes de correlação de Pearson, aferidos entre -1 e +1 , ou seja, quando os valores negativos representam correlação negativa, associação na qual diminuindo o valor para a variável, ocorre aumento nos valores do PHQ9. A correlação positiva, por sua vez, acontece quando aumentando os valores da variável, aumentam também os valores do PHQ-9. No estudo, houve variáveis cuja resposta estava condicionada a outra variável, para estas, foi calculado a medida Odds Ratio para prever a chance de um adolescente exposto a determinada condição, desenvolver sintomatologia depressiva. Estas variáveis foram adicionadas ao estudo, independente de apresentarem significância estatística, já que suas condicionantes foram significativas.

Para compreensão mais apurada dos dados, optou-se por realizar uma análise de redes, que se deu por meio do package igraph do software $\mathrm{R}$ (versão 3.3.6), considerando as possíveis interações entre todas as variáveis, e assim, identificar como estavam direta ou indiretamente vinculadas.

Hevey (2018) esclarece que a análise de redes tem sido muito utilizada em diversas áreas do conhecimento, principalmente na área da saúde para auxílio na elucidação de elementos sociais, morbidades, fatores de risco e de proteção. O autor elucida ainda que este método possibilita também encontrar novos caminhos nos sistemas, onde grupos de variáveis são densamente interconectados entre si, de maneira a fornecer informações que outras abordagens estatísticas não conseguem proporcionar.

Em uma rede as várias são representadas por nós, e a conexão entre elas por arestas. Há que se considerar o tamanho dos nós, bem como a espessura das arestas. Neste estudo, a cor verde representa conexão positiva e a cor vermelha, conexão negativa. Utilizou-se de um modelo de rede ponderada, indireta, acíclica e centralizada. Ponderada, pois as arestas representam a relação entre os nós e a magnitude dessa relação; indireta, porque analisou-se somente a associação, sem analisar a causalidade; acíclica, pois representa vários caminhos de conexão entre as variáveis, e centralizada porque há um nó central.

Análise da estrutura de comunidade foi aplicada à rede em estudo, nessas comunidades, grupos de variáveis são interconectadas entre si por apresentarem maior intensidade de relação. A rede obtida permitiu estabelecer uma relação de dependência entre as variáveis, de modo que ao modificar uma ou mais variáveis envolvidas, as outras também foram impactadas, o que ocorreu tanto em uma comunidade específica como na rede como um todo.

O estudo foi aprovado pelo Comitê Permanente de Ética em Pesquisa com seres humanos da Universidade Estadual de Maringá (COPEP/UEM) de acordo com parecer 3.764.136/2019.

\section{Resultados}

O estudo mostrou uma prevalência de $64,2 \%$ de sintomas de depressão moderada a grave nos adolescentes pesquisados, sendo 20,1\% sintomas de depressão grave. Adolescentes do sexo feminino apresentaram maior prevalência de sintomas depressivos $(72,0 \%)$ em comparação com os do sexo masculino $(43,0 \%)$. 
A idade média dos participantes foi de 15,2 anos, sendo que as mulheres representaram o maior número de participantes $(67,6 \%)$. A cor/raça prevalente foi branca $(46,9 \%)$, e a maioria referiu possuir casa própria $(63,3 \%)$ e residir com os pais $(66,1 \%)$. A renda aproximada mais frequente foi entre $\mathrm{R} \$ 1.000,00$ a $\mathrm{R} \$ 2.000,00(32,40 \%)$ (Tabela 2).

Tabela 2: Variáveis sociodemográficas dos adolescentes entrevistados que frequentam escolas em um município do Noroeste do Paraná, Brasil.

\begin{tabular}{llll}
\hline & Variável & $\mathbf{N}$ & $\mathbf{( \% )}$ \\
\hline Idade (anos) & $\mathbf{1 3}$ & 35 & 10,8 \\
& $\mathbf{1 4}$ & 68 & 21,0 \\
& $\mathbf{1 5}$ & 82 & 25,3 \\
& $\mathbf{1 6}$ & 69 & 21,3 \\
& $\mathbf{1 7}$ & 70 & 21,6
\end{tabular}

\section{Gênero}

$\begin{array}{ccc}\text { Masculino } & 105 & 32,4 \\ \text { Feminino } & 219 & 67,6\end{array}$

Cor/raça

$\begin{array}{ccc}\text { Branco } & 152 & 46,9 \\ \text { Negro } & 27 & 8,33 \\ \text { Pardo } & 123 & 38,0 \\ \text { Amarelo } & 14 & 4,3 \\ \text { Indígena } & 2 & 0,6 \\ \text { Não respondeu } & 6 & 1,8\end{array}$

Moradia

$\begin{array}{lcc}\text { Casa própria } & 205 & 63,3 \\ \text { Casa alugada } & 78 & 24,1 \\ \text { ropriedade rural } & 31 & 9,6 \\ \text { Não responderam } & 10 & 3,1\end{array}$

Mora com os pais

$\begin{array}{lll}\text { Sim } & 214 & 66,1 \\ \text { Não } & 110 & 33,9\end{array}$

Renda mensal média

\begin{tabular}{ccc} 
Menos de R\$ 1000,00 & 26 & 8,02 \\
$\mathbf{R} \$ \mathbf{1 0 0 0}$ a R\$ 2000,00 & 105 & 32,4 \\
$\mathbf{R} \$ \mathbf{2 0 0 0 , 0 0 0}$ a R\$ 3000,00 & 87 & 26,9 \\
$\mathbf{R} \$ \mathbf{3 0 0 0 , 0 0}$ a R\$ 4000,00 & 46 & 14,2 \\
Mais de R\$ 4000,000 & 43 & 13,3 \\
Não responderam & 17 & 5,2 \\
\hline
\end{tabular}

Fonte: Dados da pesquisa. 
Considerando o ponto de corte no score do $P H Q-9 \geq 10,64,2 \%$ dos adolescentes avaliados foram caracterizados com sintomas de depressão moderada a grave; 20,1\% apresentaram sintomas de depressão grave (PHQ-9 $\geq 20$ ); 20,1\% sintomas de depressão moderadamente grave (PHQ-9 $\geq 15$ ); 24,1\% sintomas de depressão moderada (PHQ-9 $\geq 10$ ); 20,1\% sintomas de

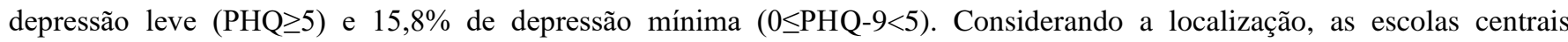
apresentaram $66,6 \%$ de alunos caracterizados com sintomas de depressão moderada à grave; escolas periféricas $67,6 \%$ e escolas do campo $23,8 \%$ (Tabela 3 ).

Tabela 3: Resultado do rastreio na população estudada, de acordo com a pontuação do PHQ-9 relacionado com a localização geográfica da escola.

\begin{tabular}{|c|c|c|c|c|c|}
\hline \multirow{2}{*}{ Pontuação } & \multirow{2}{*}{ Classificação } & \multicolumn{3}{|c|}{ Tipo de Escola (Prevalência) } & \multirow{2}{*}{$\begin{array}{c}\text { Total } \\
\text { (Prevalência) }\end{array}$} \\
\hline & & Centro & Periferia & Campo & \\
\hline $1-4$ & Depressão mínima & $24(12,30 \%)$ & $15(13,90 \%)$ & $12(57,14 \%)$ & $51(15,75 \%)$ \\
\hline $5-9$ & Depressão leve & $41(21,02 \%)$ & $20(18,50 \%)$ & $4(19,05 \%)$ & $65(20,05 \%)$ \\
\hline $10-14$ & Depressão moderada & $53(27,18 \%)$ & $23(21,30 \%)$ & $2(9,52 \%)$ & $78(24,10 \%)$ \\
\hline 15-19 & $\begin{array}{c}\text { Depressão moderadamente } \\
\text { grave }\end{array}$ & $37(19,00 \%)$ & $27(25,00 \%)$ & $1(4,77 \%)$ & $65(20,05 \%)$ \\
\hline 20-27 & Depressão grave & $40(20,50 \%)$ & $23(21,30 \%)$ & $2(9,52 \%)$ & $65(20,05 \%)$ \\
\hline Total & & $195(100 \%)$ & $108(100 \%)$ & $21(100 \%)$ & $324(100 \%)$ \\
\hline
\end{tabular}

Fonte: Dados da pesquisa.

Não havendo problemas de multicolinearidade entre as variáveis (VIF: 7,710151), sete variáveis destacaram-se como as que melhor explicam o estudo, sendo elas: sexo; tempo de uso de celular; frequentar parques; praticar esportes; usar drogas lícitas; relatar diagnóstico de ansiedade e depressão, e relatar casos de depressão na família (Tabela 4).

Tabela 4: Variáveis preditivas obtidas no estudo.

\begin{tabular}{|c|c|c|c|}
\hline Variável preditiva & $\mathrm{p}$ value $(\mathrm{p} \leq 0,05)$ & Odds ratio & IC \\
\hline Sexo & $1.16 \mathrm{e}^{-05}$ & & \\
\hline Tempo de uso de celular & 0.005088 & & \\
\hline Jogar no celular em grupo & & 0,46 & $0,25-0,81$ \\
\hline Jogar no celular sozinho & & 5,10 & $2,95-9,69$ \\
\hline Razão: sozinho/em grupo & & 11,08 & \\
\hline Frequentar parques & $6.03 \mathrm{e}^{-05}$ & & \\
\hline Praticar esportes & 0.018414 & & \\
\hline Usar drogas & 0.047470 & & \\
\hline Em grupo & & 5,54 & $1,82-16,45$ \\
\hline Sozinho & & 0,18 & $0,05-0,53$ \\
\hline Razão: em grupo/sozinho & & 30,0 & \\
\hline Relatar diagnóstico de ansiedade & 0.013767 & & \\
\hline Relatar depressão na família & 0.016900 & & \\
\hline
\end{tabular}

Fonte: Dados da pesquisa. 
Das variáveis identificadas no estudo, observou-se que, sexo (masculino), frequentar parques e praticar esportes correlacionam-se negativamente com a variável dependente PHQ-9, portanto estão associados à uma redução dos valores do PHQ-9 (Figura 1A).

As variáveis sexo (feminino); aumento do tempo de uso diário de celular; usar drogas lícitas tais como álcool, tabaco e narguilé; relatar diagnóstico de ansiedade e ter caso de depressão na família, estão correlacionadas positivamente com a variável dependente PHQ-9, portanto, consideradas fatores de risco, pois estão associadas a um aumento dos valores do PHQ-9 (Figura 1A).

Quanto às variáveis condicionais, jogar no celular sozinho está condicionada ao tempo de uso de celular, assim como usar drogas lícitas em grupo está condicionada a usar drogas lícitas, e estas variáveis associaram-se a um aumento do valor do PHQ-9 (Figura 1B). Portanto, o adolescente que usa drogas em grupo tem 5,5 vezes mais chances de desenvolver sintomas de depressão, e quando faz uso sozinho, 30,0 vezes mais chances. Um adolescente que joga no celular sozinho tem 5,1 vezes mais chances de desenvolver sintomas de depressão, e quando joga em grupo apresenta 11,1 vezes mais chances (Tabela 4). Estes resultados mostram, portanto, que o desenvolvimento de sintomas depressivos em relação ao uso de drogas sozinho ou em grupo, assim como jogar no celular sozinho ou grupo depende de sua variável condicionante.

Figura 1: Matriz de correlação das variáveis preditivas (A) e das variáveis condicionais (B) de acordo com análise de correlação de Pearson.
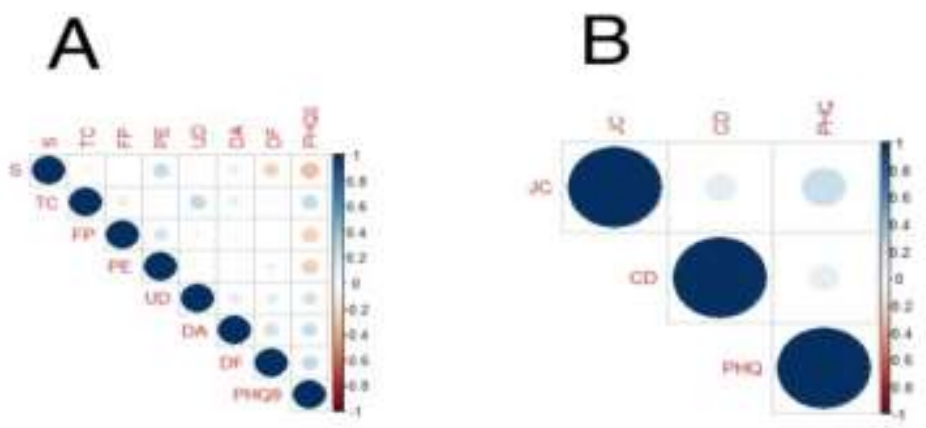

Bolas vermelhas representam correlação negativa, Bolas azuis, correlação positiva. (S) Sexo, (FP) frequentar parques, (PE) prática de esportes, (TC) tempo de uso de celular, (UD) usar drogas lícitas (álcool, tabaco e narguilé), (DA) ter sido diagnosticado com ansiedade, (DF) ter caso de depressão na família. (JC) jogar no celular, (CD) consumo de drogas. Fonte: Dados da pesquisa.

A análise de redes foi realizada, e a rede obtida classificada como acíclica, ponderada e centralizada no nó PHQ-9, partindo deste nó, são estabelecidas as demais conexões por meio das arestas. As conexões positivas mais fortes foram encontradas entre o aumento dos valores do PHQ-9 com relatar diagnóstico de ansiedade (DA); o aumento do tempo de uso diário de celular (TC) com uso de drogas (UD); relatar depressão na família (DF) com relatar diagnóstico de ansiedade (DA), e ser do sexo masculino (S) com praticar esportes (PE). As conexões negativas mais fortes foram encontradas entre PHQ-9 e ser do sexo feminino (S); entre PHQ-9 com não frequentar parques e áreas de esportes e lazer (FP), e ser do sexo feminino com relatar depressão na família (DF) (Figura 2).

Observou-se três comunidades na rede obtida: a comunidade 1 (vermelho) envolve as variáveis frequentar parques (FP), praticar esportes (PE), ser do sexo feminino (S) e PHQ9; a comunidade 2 (verde) envolve, relatar diagnóstico de ansiedade (DA) e depressão na família (DF); a comunidade 3 (azul) envolve tempo de uso diário de celular (TC) e uso de drogas lícitas (UD). 
Figura 2: Análise de rede demonstrando a correlação entre as variáveis preditivas e o diagnóstico de regressão.

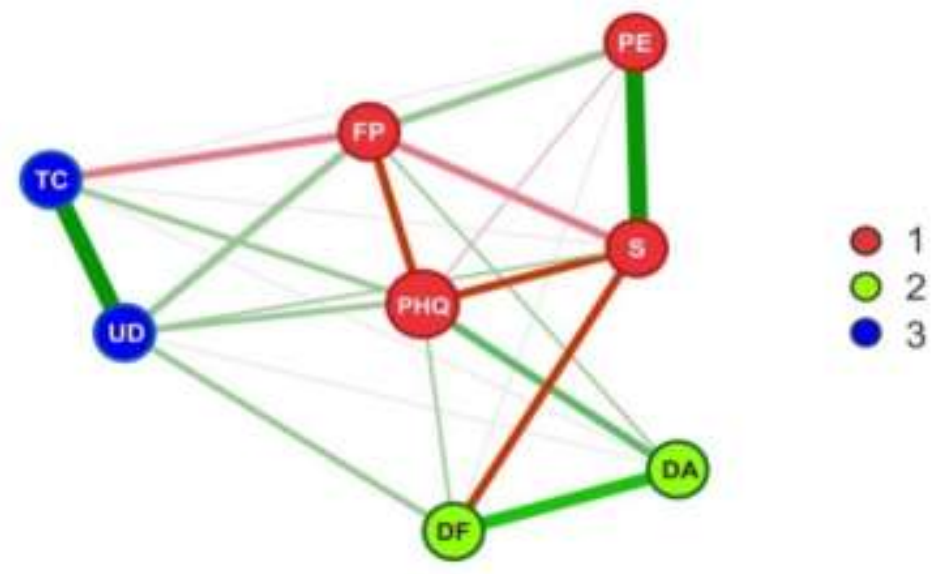

(S) Sexo, (FP) frequentar parques, (PE) prática de esportes, (TC) tempo de uso de celular, (UD) usar drogas lícitas (álcool, tabaco e narguilé), (DA) relatar diagnóstico de ansiedade, (DF) ter caso de depressão na família, (PHQ9). A figura mostra também as comunidades geradas pelas variáveis com maior relação entre si. Em vermelho, comunidade 1: FP, PE, S e PHQ9, em verde, comunidade 2: DA e DF e em azul, comunidade 3: TC e UD. Fonte: Dados da pesquisa.

\section{Discussão}

No Brasil, estudos com diferentes instrumentos de avaliação registraram taxas de prevalência de 10,2\% (Flieitlich, et al., 2004) a 13,2\% (La Maison, 2018) de sintomas de depressão em adolescentes, porém, sem considerar as especificidades do porte da cidade e a relação subjetiva de seus habitantes com o espaço geográfico e social local. Segundo a Sociedade Brasileira de Pediatria (SBP), a prevalência real da depressão nessa população ainda é desconhecida no Brasil, e estima que 59,9\% desta população tenha sintomas depressivos. Neste estudo, os dados mostraram prevalência de 64,2\% para sintomas depressivos de um modo geral (PHQ-9 $\geq 10$ ), e 20,1\% para sintomas de depressão grave (PHQ-9 $\geq 20$ ) nos adolescentes estudados.

Esta pesquisa corrobora com a maioria dos estudos, ao identificar maior prevalência de sintomas depressivos no sexo feminino quando comparado com o masculino (Machado et al., 2018) o que sugere um problema crescente de internalização nas adolescentes (Pullmer et al., 2019), associado especialmente com a maior prevalência também de transtorno de ansiedade (NING et al., 2020).

Para Park, et al. (2020) é importante destacar que os adolescentes da atualidade têm uma importante influência digital, uma vez que iniciaram o uso de aparelhos celulares precocemente, com consequente remodelamento do seu comportamento social e podem indicar que o vício em celulares e depressão se prevê simultaneamente. Neste sentido, Bickhan, Hswen e Rich (2015), encontraram associação significativa entre o telefone celular e sintomas depressivos, resultado este semelhante ao encontrado por este estudo, o que sugere, segundo os autores que os participantes ativavam sua rede social para lidar com emoções negativas. Twenge e Campbell (2018) mostraram que adolescentes usuários altos de telas (+7 h/dia) quando comparado com usuários baixos (1h/dia), tiveram duas vezes mais chances de serem diagnosticados com depressão, evidenciando a associação entre o maior tempo diário de tela de celular e menor bem estar psicológico.

Destaca-se também a alta prevalência de sintomas depressivos entre adolescentes que fazem uso de celular para jogos eletrônicos, revelando que os que jogam sozinhos apresentam maior chance de desenvolver depressão do que os que jogam em grupo, o que sugere uma associação positiva de sintomas depressivos com o fator solidão. Na, et al. (2017) em um estudo sobre o transtorno de jogo na internet mostraram que este transtorno tem sido o foco da atenção clínica no campo da saúde mental, e que os usuários demonstraram altos níveis de ansiedade social e impulsividade. este resultado é ratificado por King et 
al. (2019) ao concluírem que o tipo de jogo proporciona sintomas desadaptativos entre jogadores e jogos, gerando ansiedade e impulsividade.

Para Stein, et al. (2010) e Perez, et al. (2020), existem evidências de que o entorno social no qual o sujeito se constitui afeta diretamente a forma e a intensidade como ele expressa seus sintomas. Deste modo, o presente estudo evidenciou uma maior prevalência na periferia $(67,6 \%)$, possivelmente expressa pela vulnerabilidade social, e uma menor prevalência na área rural (23,8\%). Raphaelli, et al. (2020) em um estudo com alunos de escolas rurais concluíram que 82,3\% eram trabalhadores atuais, com atividades predominante domésticas $(39,8 \%)$ e agricultura $(29,9 \%)$, e entre os adolescentes que trabalham na agricultura familiar, o consumo de tabaco e o uso de álcool são também comportamentos preocupantes, frequentemente encontrados em residentes da zona rural.

A nova paisagem em países que sofrem transformações urbanas tem um impacto profundo na carga global da depressão (FU, 2018), impactando diretamente a vida dos adolescentes. Assim, este estudo aponta para associação positiva entre não frequentar parques e áreas de convivência com o aumento dos sintomas depressivos. Vanaken e Danckaerts (2018) apontam a relação benéfica destes espaços com o bem-estar mental em adolescentes e Dzambov (2018) esclarece que estes locais estão associados a uma melhor saúde mental.

Para Min, et al. (2017) há evidências de associação protetora entre parques e áreas verdes com indicadores de depressão e suicídio. Deste modo, e considerando a carências de espaços verdes urbanos em muitas cidades de pequeno porte, Pode-se considerar, portanto, que nestas localidades pode haver um aumento do risco para o desenvolvimento de sintomas depressivos em adolescentes.

Min et al. (2017), pontua ainda que atividade física moderada pode ajudar a diminuir o risco de depressão, ponto este, também observado por este estudo ao mostrar associação positiva entre não praticar esportes e sintomas depressivos. He, Parksarian e Merikangas (2018) mostram que adolescentes ativos têm chances significativamente menores de apresentar transtorno de humor, transtorno bipolar e sofrimento psicológico, e que os inativos e que não praticam atividade física têm maior probabilidade para desenvolver transtorno de uso de álcool, bulimia, transtorno de ansiedade generalizada e transtorno de estresse pós-traumático ao longo da vida. Além disso, a prática de atividades físicas promove benefícios psicológicos e sociais à saúde, como autoestima mais aprimorada e interação social, seguida por menos sintomas depressivos (Brito et al., 2019).

A análise de redes deste estudo apontou conexão positiva entre ser do sexo masculino e frequentar parques, indicando que estes espaços são frequentados especialmente por homens. Para as mulheres, resta-lhes poucas opções, sendo, portanto, a ausência de local apropriado para a atividade um dos fatores que implicam na falta de atividade física regular para esta população, como esclarecido por Gomes, et al. (2019). Desta forma, este estudo chama a atenção para a possibilidade de que cidades de pequeno porte tenham poucos espaços para a prática de esportes, e ainda assim, quando possuem, são atividades voltadas especialmente para o sexo masculino. Os locais que oferecem atividade física para as mulheres são, na sua maioria, estabelecimentos pagos, o que dificulta o acesso a estes espaços.

Uso de drogas lícitas como álcool, tabaco e narguilé é considerado um importante fator de risco para desenvolvimento de sintomas depressivos em adolescentes. Marin, et al. (2019), encontraram forte associação entre álcool e depressão, corroborando com os achados desse estudo. Por outro lado, para Bai, et al. (2018) reduzir o fumo e uso de substâncias semelhantes por adolescentes, diminuem os sintomas depressivos e aumentam a satisfação com os cuidados de saúde mental.

Um dos fatores de risco para depressão mais comum encontrado em adolescentes é o transtorno de ansiedade. Segundo La Maison, (2018), a ansiedade, expressa no início da vida, é seguida pela depressão na idade adulta, e para Jatobá e Bastos (2007), 40\% dos transtornos depressivos em adolescentes associam-se ao transtorno de ansiedade. Orellana, et al. (2020), elucidam que a ocorrência de transtornos de ansiedade e depressão costuma estar associada à maior gravidade desses 
quadros, menor resposta ou abandono do tratamento, além de oferecerem risco adicional de suicídio, e que em geral, os transtornos de ansiedade apresentam prevalência notadamente superior nas mulheres, independentemente do local e da idade, como também observado por este estudo.

Ter casos de depressão na família revela o quanto componentes genéticos, ambientais e familiares podem interferir no desenvolvimento dos adolescentes. Para Gorostiaga (2019), o "calor" dos pais, o controle comportamental e a concessão de autonomia estão inversamente relacionados aos sintomas internalizantes em adolescentes, e o controle psicológico severo estão associados positivamente à ansiedade, depressão e ideação suicida.

Mullins e Lewis esclarecem que a depressão é um distúrbio complexo, com herdabilidade de 37\%, e apesar das evidências robustas de um componente genético, identificar as variantes genéticas específicas envolvidas é um grande desafio. Deste modo, evidenciou-se, neste estudo, que casos de depressão familiar impactam o desenvolvimento de sintomas depressivos em adolescentes.

Yun et al. (2019), em um estudo com adolescentes coreanos constataram que pais e professores muitas vezes não reconhecem os primeiros sinais de depressão em adolescentes, o que dificulta o acesso dos adolescentes deprimidos aos serviços de saúde e consequente ao tratamento. De acordo com Malas, Plioplys e Pao (2019), embora a terapia medicamentosa não seja a única possível para tratar adolescentes deprimidos, é considerada como marco para avaliar a porcentagem dos adolescentes em tratamento, e mesmo que a maioria dos jovens medicamente doentes sejam resilientes e estejam bem, os efeitos da doença podem piorar o funcionamento diário, a autoestima, o humor e a qualidade de vida, para os autores, o atendimento psiquiátrico é necessário quando um paciente apresenta depressão recorrente, é resistente ao tratamento ou apresenta desregulação comportamental significativa, incluindo não adesão ao tratamento.

\section{Conclusão}

$\mathrm{O}$ aumento de sintomas depressivos em adolescentes inspira cuidados. Entender o contexto em que este agravo se estabelece se faz necessário para melhor cuidar. Deste modo, buscou-se elucidar os fatores de risco associados aos sintomas de depressão em adolescentes que vivem em cidades pequenas. Os resultados apontaram que o maior tempo de uso diário de celular despendido em jogos eletrônicos; não frequentar parques e locais de convivência; não praticar esportes; usar drogas lícitas como tabaco, álcool e narguilé; relatar diagnóstico para ansiedade; relatar casos de depressão na família e ser do sexo feminino são fatores que tiveram uma associação estatisticamente significativa com sintomas depressivos em adolescentes.

Deste modo, em se tratando de adolescentes que vivem em cidades pequenas, pode-se dizer que há uma falta de estrutura adequada que promova melhores perspectivas de futuro, assim como possibilidades reais de ser e realizar-se completamente. Não sendo acolhedoras, principalmente para as adolescentes, estas cidades deixam de contribuir para uma boa saúde mental. Deste modo, se faz urgentemente necessário promover os espaços públicos e privados à espaços mentalmente saudáveis, proporcionar acompanhamento multiprofissional para um correto diagnóstico, bem como melhorar o acesso ao sistema público de saúde mental por meio de políticas inclusivas e acolhedoras para adolescentes.

As principais limitações deste estudo estão relacionadas ao fato de a pesquisa contemplar somente adolescentes estudantes, embora a amostra analisada proporcione uma boa visão da população. Também não foi realiza a repetição do teste para averiguação dos resultados.

Sugere-se, como trabalhos futuros, que sejam feitos estudos com uma amostra mais abrangente da população adolescente envolvendo não somente estudantes, mas adolescentes que não estudam e que estão em situações de risco. 


\section{Referências}

American Psychiatric Association. Diagnostic and Statistical Manual of Mental Disorders, Fifth Edition (DSM-V). Arlington, VA: American Psychiatric Association, 2013.

Bai, S., Zeledon, L. R., D'Amico, E. J., Shoptaw, S., Avina, C., LaBorde, A. P., Anderson, M., Fitzpatrick, O. M., \& Asarnow, J. R. (2018). Reducing Health Risk Behaviors and Improving Depression in Adolescents: A Randomized Controlled Trial in Primary Care Clinics. Journal of pediatric psychology, 43(9), 1004-1016. https://doi.org/10.1093/jpepsy/jsy048

Bickham, D. S., Hswen, Y., \& Rich, M. (2015). Media use and depression: exposure, household rules, and symptoms among young adolescents in the USA. International journal of public health, 60(2), 147-155. https://doi.org/10.1007/s00038-014-0647-6

Brazilian Society of Pediatrics (SBP). Brazil. 2019. https://www.sbp.com.br/imprensa/detalhe/nid/aumento-da-depressao-na-infancia-e-adolescenciapreocupa-pediatras/

Brito, M. F. S. F., Pinho, L., Brito, A. B., Messias, R. B., Pinto, S. Oliveira, A. A., Silva, C. S. O., Volker, V., \& Silveira, M. F. (2019). Fatores associados ao estilo de vida de estudantes do ensino médio de escolas públicas. Rev Gaúcha Enferm. 40:e20180168. https://doi. org/10.1590/1983-1447.2019.20180168.

David Hevey (2018) Network analysis: a brief overview and tutorial, Health Psychology and Behavioral Medicine, 6:1, 301-328, $10.1080 / 21642850.2018 .1521283$

Dzhambov, A. M. (2018). Residential green and blue space associated with better mental health: a pilot follow-up study in university students. Arhiv za higijenu rada i toksikologiju, 69(4), 340-349. https://doi.org/10.2478/aiht-2018-69-3166

Fatori, D., et al (2018). Prevalência de problemas de saúde mental na infância na atenção primária. Ciência \& Saúde Coletiva,23(9), 30133020. https://doi.org/10.1590/1413-81232018239.25332016

Fleitlich-Bilyk, B., \& Goodman, R. (2004). Prevalence of child and adolescent psychiatric disorders in southeast Brazil. Journal of the American Academy of Child and Adolescent Psychiatry, 43(6), 727-734. https://doi.org/10.1097/01.chi.0000120021.14101.ca

Fu, Q. (2018). Communal space and depression: A structural-equation analysis of relational and psycho-spatial pathways. Health \& Place. Sep; 53: 1-9. DOI: 10.1016/j.healthplace.2018.06.007.

Gomes, G. A. de O., Papini, C. B., Nakamura, P. M., Teixeira, I. P., \& Kokubun, Eduardo. (2019). Barreiras para prática de atividade física entre mulheres atendidas na Atenção Básica de Saúde. Revista Brasileira de Ciências do Esporte, 41(3), 263-270. 2019.https://doi.org/10.1016/j.rbce.2018.04.007

Gorostiaga, A., Aliri, J., Balluerka, N., \& Lameirinhas, J. (2019). Parenting Styles and Internalizing Symptoms in Adolescence: A Systematic Literature Review. International journal of environmental research and public health, 16(17), 3192. https://doi.org/10.3390/ijerph16173192

He, J. P., Paksarian, D., \& Merikangas, K. R. (2018). Physical Activity and Mental Disorder Among Adolescents in the United States. The Journal of adolescent health: official publication of the Society for Adolescent Medicine, 63(5), 628-635. https://doi.org/10.1016/j.jadohealth.2018.05.030

Instituto Brasileiro de Geografia e Estatística (IBGE cidades) page. https://cidades.ibge.gov.br/.

Instituto Brasileiro de Geografia e Estatística (IBGE) page. https://censo2010.ibge.gov.br/sinopse/index.php?uf=41\&dados=6

Instruction manual for the Patient Health Questionaire (PHQ-9) and CAD-7. www.phqscreeners.com

Jatobá, J. D’Arc V.N., \& Bastos, Othon. (2007). Depressão e ansiedade em adolescentes de escolas públicas e privadas. Jornal Brasileiro de Psiquiatria, 56(3), 171-179. https://dx.doi.org/10.1590/S0047-20852007000300003

Johnson, R. A., \& Wichern, D. W. (2007). Applied multivariate statistical analysis, Madison: Prentice Hall International.

King, D. L., Delfabbro, P. H., Perales, J. C., Deleuze, J., Király, O., Krossbakken, E., \& Billieux, J. (2019). Maladaptive player-game relationships in problematic gaming and gaming disorder: A systematic review. Clinical psychology review, 73, 101777. https://doi.org/10.1016/j.cpr.2019.101777

La Maison, C., Munhoz, T. N., Santos, I. S., Anselmi, L., Barros, F. C., \& Matijasevich, A. (2018). Prevalence and risk factors of psychiatric disorders in early adolescence: 2004 Pelotas (Brazil) birth cohort. Social psychiatry and psychiatric epidemiology, 53(7), 685-697. https://doi.org/10.1007/s00127-018-1516-Z

Machado, I.C., Silva, N.S.S., Haikal, D.S., Silveira, M.F., Alves, M.L.T.S., \& Silva, R.R.V. (2018). Prevalência De Sintomas Depressivos Entre Adolescentes Da Rede Pública De Ensino. Adolesc. Saúde. 1(4): 27-35.

Malas, N., Plioplys, S., \& Pao, M. (2019). Depression in Medically Ill Children and Adolescents. Child and adolescent psychiatric clinics of North America, 28(3), 421-445. https://doi.org/10.1016/j.chc.2019.02.005

Marin, A. H., Peuker, A. C., \& Kessler, F. H. P. (2019). Sociodemographic Characteristics, School Performance, Pattern of Consumption and Emotional Health as Risk Factors for Alcohol use among Adolescents. Trends in Psychology, 27(1), 279-292. https://doi.org/10.9788/tp2019.1-20

Min, K.b., Kim, H.J., Kim, H.J., \& Min, J.Y. (2017). Parks and green areas and the risk for depression and suicidal indicators. Int J Public Health 62, 647656. https://doi.org/10.1007/s00038-017-0958-5

Mullins, N., \& Lewis, C. M. (2017). Genetics of Depression: Progress at Last. Current psychiatry reports, 19(8), 43. https://doi.org/10.1007/s11920-017-08039

Na, E., Choi, I., Lee, T. H., Lee, H., Rho, M. J., Cho, H., Jung, D. J., \& Kim, D. J. (2017). The influence of game genre on Internet gaming disorder. Journal of behavioral addictions, 6(2), 1-8. Advance online publication. https://doi.org/10.1556/2006.6.2017.033 
Research, Society and Development, v. 10, n. 4, e49210414315, 2021

(CC BY 4.0) | ISSN 2525-3409 | DOI: http://dx.doi.org/10.33448/rsd-v10i4.14315

Ning, K., Gondek, D., Patalay, P., \&Ploubidis, G.B. (2020). The association between early life mental health and alcohol use behaviours in adulthood: A systematic review. PLOS ONE. 15(2): e0228667. https://doi.org/10.1371/journal.pone.0228667

Orellana, J. D. Y., Ribeiro, M. R. C., Barbieri, M. A., Saraiva, M. da C., Cardoso, V. C., Bettiol, H. Silva, A. A. M. da, Barros, F. C., Gonçalves, H., Wehrmeister, F. C., Menezes, A. M. B., Del-Ben, C. M., \& Horta, B. L. (2020). Transtornos mentais em adolescentes, jovens e adultos do Consórcio de Coortes de Nascimento brasileiras RPS (Ribeirão Preto, Pelotas e São Luís). Cadernos de Saúde Pública, 36(2), e00154319. 31, 2020.https://doi.org/10.1590/0102-311x00154319

Park, S. Y., Yang, S., Shin, C. S., Jang, H., \& Park, S. Y. (2019). Long-Term Symptoms of Mobile Phone Use on Mobile Phone Addiction and Depression Among Korean Adolescents. International journal of environmental research and public health, 16(19), 3584. https://doi.org/10.3390/ijerph16193584

Pereira A. S. et al. (2018). Metodologia da pesquisa científica. UFSM.

Pérez, C. A., Valdés, M. E. F., Ramos, K. F., González, L. I. F., \& Figueredo, I. C. G. (2020) Indicadores de la realidad de un grupo de púberes en el entorno escolar. Multimed, 24(6), 1258-1270. http://scielo.sld.cu/scielo.php?script=sci_arttext\&pid=S1028-48182020000601258\&lng=es\&tlng=es.

Pullmer, R., Chung, J., Samson, L., Balanji, S., \& Zaitsoff S. (2019) A systematic review of the relation between self-compassion and depressive symptoms in adolescents. Journal of Adolescence74:210-220. 10.1016/j.adolescence.2019.06.006.

Raphaelli, C. de O., et al (2020). Cross-sectional study on work and health risk behaviors among school students in a rural area of Rio Grande do Sul State, Brazil, 2010. Epidemiologia e Serviços de Saúde, 29(3), e2019285. https://doi.org/10.5123/s1679-49742020000300006

Stein, G., Curry, J., Hersh, J., Breland-Noble, A., March, J., Silva, S., Reinecke, M., \& Jacobs, R. (2010). Ethnic Differences Among Adolescents Beginning Treatment for Depression. Cultural diversity \& ethnic minority psychology. 16. 152-8. 10.1037/a0018666.

Twenge J.M., \& Campbell, K. (2018) Associations between screen time and lower psychological well-being among children and adolescents: Evidence from a population-based study. Preventive Medicine Reports. 12: 271-283

Vanaken, G. J., \& Danckaerts, M. (2018). Impact of Green Space Exposure on Children's and Adolescents' Mental Health: A Systematic Review. International journal of environmental research and public health, 15(12), 2668. https://doi.org/10.3390/ijerph15122668

World Health Organization (WHO), 2018. https://nacoesunidas.org/oms-1-em-cada-5-adolescentes-enfrenta-problemas-de-saude-mental/

World Health Organization (WHO), 2020. Depression. https://www.who.int/health-topics/depression\#tab=tab_1.

Yun, J. Y., Chung, H., Sim, J. A., \& Yun, Y. H. (2019) Prevalence and associated factors of depression among Korean adolescents. PLoS ONE 14(10): e0223176. https://doi.org/10.1371/journal.pone.0223176 